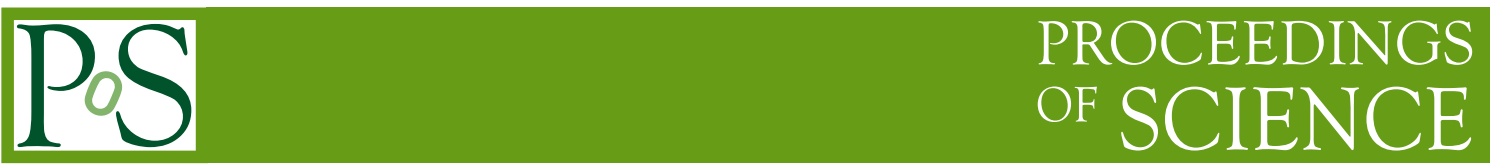

\title{
The in-flight calibration of the TUS orbital detector
}

\author{
L.Tkachev* \\ Joint Institute for Nuclear Research, Dubna, Russia \\ E-mail: tkatchev@jinr.ru \\ V.Boreiko, V.Grebenyuk, N.Gorbunov, B.Sabirov \\ Joint Institute for Nuclear Research, Dubna, Russia
}

A.Tkachenko

Joint Institute for Nuclear Research, Dubna, Russia

Bogolyubov Institute for Theoretical Physics, Kiev, Ukraine

A. Dmitroza, A.Volvach
Crimea Astrophysical Observatory, Simeiz, Russia

P. Klimov

Lomonosov Moscow State University, Skobeltsyn Institute of Nuclear Physics, Moscow, Russia

\section{E. M. Popescu}

Institute of Space Science, Magurele, Romania

The TUS space experiment is aimed to study the energy spectrum and arrival directions of Ultra High Energy Cosmic Rays (UHECR) at $\mathrm{E} \sim 10^{20} \mathrm{eV}$ by measuring the fluorescence yield of EAS in the atmosphere. The TUS mission is scheduled for launch at the end of 2015 aboard the dedicated "Lomonosov" satellite, and is expected to operate for 3-5 years. The TUS collaboration plans to design and produce a ground-based system of light sources for the in-flight calibration of the TUS detector. Two calibration methods relying on ground-based calibration sources are presented, as well as two possible prototype calibration light systems, namely high-power LEDs (e.g. LedEngin LZ4-00U600) and laser stations such as the "Simeiz-1873" laser station.

The 34th International Cosmic Ray Conference,

30 July- 6 August, 2015

The Hague, The Netherlands

\footnotetext{
*Speaker
} 


\section{Introduction}

The TUS mission is a satellite mission for the experimental study of UHECR. The fluorescence and Cherenkov UV yield of Extensive Air Showers (EAS) generated by UHECR particles will be detected at the night side of the Earth atmosphere from the orbital space platform at altitudes in the 400-500 km range. This will allow for the measurement of the CR spectrum, composition and arrival directions at $E>7 \cdot 10^{19} \mathrm{eV}$ [1], [2] beyond the GZK energy limit.

As one can see in Fig. 1 the TUS UV telescope has two main components: a modular Fresnel mirror and a $16 \times 16$ pixels photo-receiver matrix with the corresponding DAQ electronics [3]. The Fresnel mirror has an area of $1.8 \mathrm{~m}^{2}$ and a focal distance of $1.5 \mathrm{~m}$, each pixel of the photo-receiver consists of a $13 \mathrm{~mm}$ diameter multi-alcali cathode Hamamatsu R1463 PMT with UV glass window, and the DAQ electronics has 256 separate channels with a time resolution of $0.8 \mu$ s per channel. The TUS UV telescope has a total FoV of $\pm 4.5^{\circ}$, with an FoV per pixel of $\sim 0.1 \mathrm{msr}$ corresponding to a spatial resolution of $5 \times 5 \mathrm{~km}$ on the Earth surface for an orbit height of $500 \mathrm{~km}$.

At present, the production phase of the TUS UV telescope has been completed, and the telescope is currently undergoing pre-flight tests. The TUS mission is scheduled for launch at the end of 2015 aboard the dedicated "Lomonosov" satellite, and is expected to operate for $3 \div 5$ years [4]. This will be the first orbital mission for the study of the UV fluorescence yield of EASs produced by UHECR, and atmospheric effects aside, it will allow for a more stable study of EASs than ground-based observations. Moreover, it is important to emphasize that the TUS mission will provide a systematic and more uniform coverage of both the northern and southern hemispheres, it will allow for the possibility to better understand the differences between the results of the Pierre Auger Observatory in the southern hemisphere and those of the TA in the northern hemisphere.

However, as the EAS UV photon flux reaching the orbital TUS telescope is $~ 100$ times

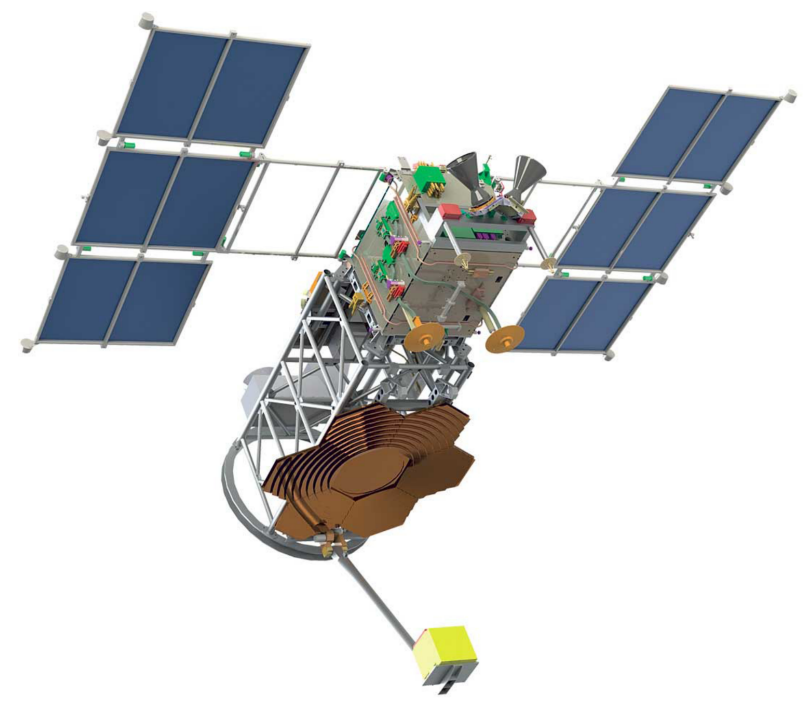

Figure 1: The TUS Detector aboard the Lomonosov satellite. 
weaker than the corresponding flux available to ground-based observatories, the issue of calibrating the telescope in the presence of night-sky background light is crucial. Under these circumstances, regular monitoring and calibration of the orbital telescope is needed during the entire lifetime of the TUS mission, in order to ensure the accuracy and reliability of the data. For this purpose, dedicated high-power LED and laser light sources will be used at appropriately chosen locations on the ground to provide periodic calibration signals to the orbital telescope. The evaluation of the operational characteristics of these light sources constitutes the topic of the present work, and our approach and results will be presented in the following sections.

\section{Calibration methods}

In this section we present the ground-based calibration methods that are currently under development for the TUS mission. These methods rely on the use of ground-based light sources illuminating the TUS telescope while in orbit to allow for the testing and correction of the telescope photo-detector and associated electronics calibration parameters on a regular basis. Two calibration methods are considered here, which are based on the indirect and direct illumination of the orbital telescope with high-power LEDs and lasers.

\subsection{Telescope calibration by indirect illumination}

In this method, a high-power LED or a laser are used to generate a pulsed light beam in the spectral range 300-400 $\mathrm{nm}$ which is shot through the atmosphere at some angle with respect to the horizon. During its propagation through the atmosphere, photons are scattered according to the Rayleigh mechanism, and some of them, after suffering subsequent scattering, reach the input aperture of the TUS telescope. For an orbital altitude of $\sim 500$ $\mathrm{km}$ and a FoV of $\pm 4.5^{\circ}$, there is a time interval of $\sim 10 \mathrm{~s}$ for the optical visibility between the light source and the telescope, allowing for the calibration of a significant number of pixels of the TUS telescope in one calibration run. However, since a valid calibration requires about $100-500$ photons $/\left(\mathrm{m}^{2} \mu \mathrm{s}\right)$ to reach each of the pixels of the telescope in orbit, this calibration method requires a relatively high-power light beam, with photon rates exceeding $10^{15}$ photons $/ \mu \mathrm{s}$.

In order to evaluate the light flux reaching the TUS telescope in orbit, consider a horizontal pulsed light beam at sea level with $N_{0}$ photons of $\lambda$ wavelength. Assuming an isothermal atmospheric model, the photon loss due to Rayleigh scattering along a path of length $d l$ in the atmosphere is:

$$
\begin{aligned}
\frac{d N}{N} & =-\frac{\rho_{0} \cdot d l}{R(\lambda)} \\
R(\lambda) & =2974\left[\mathrm{~g} / \mathrm{cm}^{2}\right]\left(\frac{\lambda}{400 \mathrm{~nm}}\right)^{4},
\end{aligned}
$$

where $\rho_{0}$ is the atmosphere density. The total number of the photons scattered away from the beam after traveling a distance $l$ will be: 


$$
\Delta N=N_{0}\left[1-\exp \left(-\frac{\rho_{0} \cdot l}{R(\lambda)}\right)\right] .
$$

The angular distribution of scattered photons is:

$$
\Phi(\theta, \phi)=\frac{3}{16 \pi}\left(1+\cos ^{2} \theta\right), \quad \int_{4 \pi} \Phi(\theta) d \Omega=1 .
$$

Let's suppose for simplicity that $\theta=\pi / 2$ for the photons reaching the TUS telescope mirror. The fraction of photons scattered in the TUS telescope solid angle $d \Omega_{m}$ is the following:

$$
\left.\int_{d \Omega_{m}} \Phi(\theta) d \Omega \approx \frac{3}{16 \pi}\left(1+\cos ^{2} \theta\right)\right|_{\theta=\frac{\pi}{2}} d \Omega_{m}=\frac{3}{16 \pi} \frac{S}{L^{2}},
$$

where $S$ is the mirror surface area and $L$ is the distance between the light beam axis and the detector orbit. Accounting for the extinction of photons along the path from the beam to the telescope finally yields:

$$
\Delta N=N_{0} \cdot k_{T} \cdot \frac{3}{16 \pi} \frac{S}{L^{2}} \cdot\left[1-\exp \left(-\frac{\rho_{0} \cdot l}{R}\right)\right] .
$$

where $k_{T}$ is the atmosphere transparency coefficient.

If the light pulse travels a distance $\mathscr{L}=n \cdot l$, then the number of the photons reaching the TUS mirror at the $i$-the segment will be:

$$
\begin{aligned}
\Delta N_{i} & =N_{0} k_{T} \frac{3}{16 \pi} \frac{S}{L^{2}}\left[1-e^{-\frac{\rho_{0} l}{R}}\right] e^{-i \cdot \frac{\rho_{0} l}{R}} \\
\Delta N_{i+1} & =\Delta N_{i} \cdot e^{-\frac{\rho_{0} l}{R}} \Rightarrow \Delta N_{i}=\Delta N_{0} \cdot(0.703)^{i},
\end{aligned}
$$

with $i=0-15$ and $\Delta N_{0}(\approx 1225)$ according to $(2.6)$. The total number of photons removed from the beam will then be:

$$
\Delta N_{\mathscr{L}}=\sum_{i=0}^{n-1} \Delta N_{i}=N_{0} k_{T} \frac{3}{16 \pi} \frac{S}{L^{2}} \cdot\left[1-\exp \left(-\frac{\rho_{0} \cdot \mathscr{L}}{R}\right)\right] .
$$

Since in our case $\rho_{0} \cdot l \ll R$, the expression in square brackets can be expanded in series, and to the first order we get:

$$
\Delta N_{i} \approx \frac{N_{0} k_{T}}{4 \pi L^{2}} \frac{d}{R_{L}} e^{-i \cdot \frac{d}{R}}, \quad d=\rho_{0} \cdot l, \quad R_{L}=\frac{4 R}{3 S} .
$$

The expression in (2.8) can be easily generalized to the case of a light beam inclined at an angle $\delta$ with respect to the horizon:

$$
\rho(h)=\rho_{0} \exp \left(-\frac{h}{h_{A}}\right), \quad h_{A}=7.1 \cdot 10^{5} \mathrm{~cm} .
$$

The depth of the path traversed by the light beam (in $\mathrm{g} / \mathrm{cm}^{2}$ ) at each step $\Delta=l / \cos \delta$ and for the full path $n \cdot \Delta$ in the atmosphere are: 


$$
\begin{gathered}
d_{i}=\rho_{i}(h) \cdot \Delta=\rho_{0} \exp \left(-\frac{h_{i}}{h_{A}}\right) \cdot \frac{l}{\cos \delta}=\rho_{0} \exp \left(-\frac{l \cdot\left(i+\frac{1}{2}\right) \tan \delta}{h_{A}}\right) \frac{l}{\cos \delta} . \\
d(\mathscr{L})=\int_{0}^{\mathscr{L}} \rho(h) d l=\frac{\rho_{0} h_{A}}{\sin \delta}\left[1-\exp \left(\frac{\mathscr{L} \tan \delta}{h_{A}}\right)\right] .
\end{gathered}
$$

In the limit $\delta \rightarrow 0, d(\mathscr{L}) \rightarrow \rho_{0} \mathscr{L}, \mathscr{L}=n \cdot l$ due to the fact that

$$
\Delta N_{i} \sim N_{i} \cdot\left[1-\exp \left(-\frac{d_{i}}{R}\right)\right] \quad \text { and } \quad N_{i+1}=N_{i} \cdot e^{-\frac{d_{i}}{R}}
$$

and therefore, it immediately follows that:

$$
\Delta N_{i}=N_{0} k_{T} \frac{3}{16 \pi} \frac{S}{L^{2}}\left[1-e^{-\frac{d_{i}}{R}}\right] \exp \left(-\frac{1}{R} \sum_{j=0}^{i-1} d_{j}\right) .
$$

The formula (2.13) clearly reduces to (2.8) for a horizontal light beam $d_{i}=\rho_{0} \cdot l$, and in the $\rho_{0} \cdot l \ll R$ approximation, it yields:

$$
\begin{gathered}
\Delta N_{i} \approx \frac{N_{0} k_{T}}{4 \pi L^{2}} \frac{d_{i}}{R_{L}} \exp \left(-\frac{1}{R} \sum_{j=0}^{i-1} d_{j}\right) . \\
\frac{1}{R} \sum_{j=0}^{i-1} d_{j}=p \cdot e^{-t / 2} \sum_{j=0}^{i-1} e^{-j t}=\left(\frac{p e^{-t / 2}}{1-e^{-t}}\right) \cdot\left[1-e^{-i t}\right]=\frac{p\left(1-e^{-i t}\right)}{2 \sinh (t / 2)} ; \\
t=\frac{l \cdot \tan \delta}{h_{A}}, \quad p=\frac{\rho_{0} \cdot l}{R \cos \delta} .
\end{gathered}
$$

From (2.11) it follows that:

$$
\begin{gathered}
\lim _{\substack{l \rightarrow 0, i \rightarrow \infty \\
i \cdot l \rightarrow \mathscr{L}}}\left(\frac{p}{2 \sinh (t / 2)}\right) \cdot\left[1-e^{-i t}\right]=\left(\frac{p}{t}\right) \cdot\left[1-\exp \left(-\frac{\mathscr{L} \tan \delta}{h_{A}}\right)\right] \\
=\frac{\rho_{0} h_{A}}{R \sin \delta}\left[1-\exp \left(-\frac{\mathscr{L} \tan \delta}{h_{A}}\right)\right]=d(\mathscr{L}) / R
\end{gathered}
$$

Putting all these results together, one obtains the following expressions for the $i^{t h}$-step and respectively for the full distance path $\mathscr{L}=n \cdot l$ :

$$
\begin{aligned}
& \Delta N_{i}=N_{0} k_{T} \frac{3}{16 \pi} \frac{S}{L^{2}}\left(1-\exp \left[-p e^{-\left(i+\frac{1}{2}\right) t}\right]\right) \exp \left(-\frac{p\left(1-e^{-i t}\right)}{2 \sinh (t / 2)}\right) \\
& \Delta N_{\mathscr{L}}=N_{0} k_{T} \frac{3}{16 \pi} \frac{S}{L^{2}}\left[1-\exp \left(-\frac{p}{2 \sinh (t / 2)}\left(1-e^{\left.-\mathscr{L} \tan \delta / h_{A}\right)}\right)\right]\right.
\end{aligned}
$$

The results of our calculations using (2.15) are presented in the Table 1 below at the wavelength $\lambda=355 \mathrm{~nm}$. 
As it can be seen from the rough evaluation in Table 1, up to 8 consecutive telescope pixels will be illuminated simultaneously by a number of photons scattered from the beam exceeding the $\sim 100$ photons threshold for a pixel. This means that if the light beam is oriented normally to the orbital plane of the telescope, this method will allow for the calibration of $8 \times 16$ pixels in one calibration run.

\subsection{Telescope calibration by direct illumination}

In this method, a light beam generated by high-power LED system is shot vertically in the FoV of the TUS telescope, directly illuminating its pixels. In this way, the image of the calibration source can cross up to 16 adjacent telescope pixels in one calibration run.

The calibration source parameters were evaluated based on Monte-Carlo simulations of the TUS telescope that account for the optical system and trigger electronics characteristics [5]- [8]:

1. Light pulse duration $-\geq 40 \mu \mathrm{s}$

2. The pulse energy - $10-50 \mathrm{~mJ}\left(1.9 \times 10^{16}-9.6 \times 10^{16}\right.$ photons $)$

3. The spectral interval $-300-400 \mathrm{~nm}$

4. The pulse frequency $-0.5-10 \mathrm{~Hz}$

5. Angular divergence of light beam $\pm 4.5^{\circ}$

6. Intensity of light flux at the $500 \mathrm{~km}$ latitude $100-500$ photons $/\left(\mathrm{m}^{2} \mu \mathrm{s}\right)$

7. Duration time of the calibration session - $10-100 \mathrm{sec}$

8. Long term stability of light source $1-3 \%$.

Much like in the case of the first method, the LED light source must provide 100500 photons $/\left(\mathrm{m}^{2} \mu \mathrm{s}\right)$ per pixel at the TUS telescope orbital altitude in order for the pixel calibration to be reliable. However, for both this method and the method described in the previous section have the same caveat, namely the absence of an adequate description of

\begin{tabular}{|l||c|c|c|c|c|c|c|c|c|c|c|}
\hline & $0^{\circ}$ & $2^{\circ}$ & $4^{\circ}$ & $6^{\circ}$ & $8^{\circ}$ & $10^{\circ}$ & $12^{\circ}$ & $14^{\circ}$ & $16^{\circ}$ & $18^{\circ}$ & $20^{\circ}$ \\
\hline \hline 1 & 1223 & 1211 & 1200 & 1191 & 1183 & 1176 & 1170 & 1165 & 1161 & 1159 & 1157 \\
2 & 860 & 837 & 816 & 795 & 775 & 756 & 737 & 719 & 701 & 684 & 667 \\
3 & 604 & 584 & 563 & 543 & 524 & 504 & 485 & 466 & 447 & 429 & 411 \\
4 & 425 & 410 & 395 & 380 & 364 & 347 & 331 & 315 & 299 & 282 & 267 \\
5 & 298 & 291 & 281 & 271 & 259 & 246 & 234 & 220 & 207 & 193 & 180 \\
6 & 210 & 207 & 203 & 196 & 188 & 179 & 169 & 159 & 148 & 136 & 125 \\
7 & 147 & 149 & 148 & 145 & 140 & 133 & 126 & 117 & 108 & 99 & 89 \\
8 & 103 & 108 & 110 & 109 & 106 & 101 & 95 & 88 & 80 & 73 & 65 \\
9 & 73 & 79 & 82 & 83 & 82 & 78 & 73 & 67 & 61 & 54 & 48 \\
10 & 51 & 58 & 62 & 64 & 64 & 61 & 57 & 52 & 47 & 41 & 35 \\
\hline
\end{tabular}

Table 1: The expected number of photons in consecutive telescope pixels as a function of the light beam inclination angle according to (2.15). 
photon extinction fluctuations along the path from the ground source to the telescope in orbit.

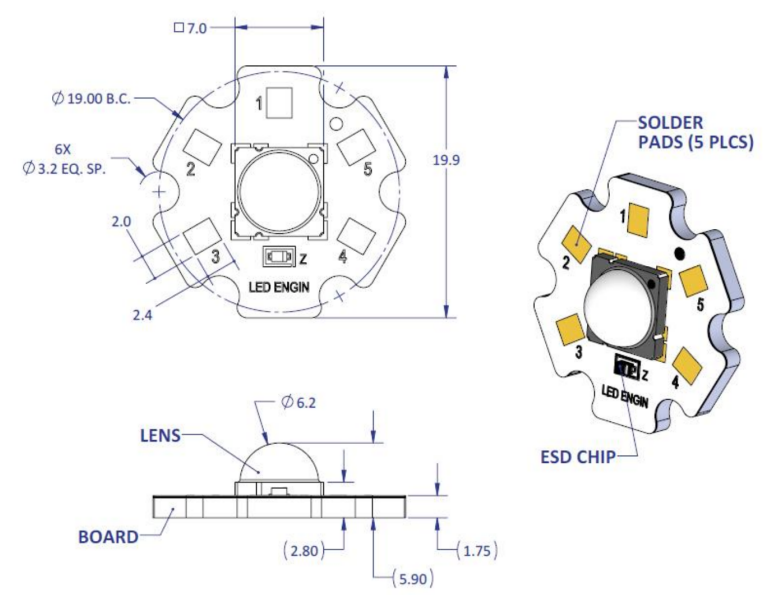

Figure 2: Schematic representation of the LedEngin-LZ4-00U600 LED together with the solder pad board and lens

\section{LED prototype source for the TUS telescope calibration}

The design and fabrication of prototype LED sources for the TUS telescope calibration through direct and indirect illumination is presently under way both at JINR and ISS. The LED of choice for the development of the light source is the LedEngin Inc. LZ4-00U600 LED [9], shown schematically in Fig. 2. It is a compact $7 \mathrm{~mm} \times 7 \mathrm{~mm}$ high-power UV LED (11 W optical output power), with the optical output spectrum confined to a narrow band around $\lambda=365 \mathrm{~nm}$ and with an integrated glass lens, which make it a suitable candidate for a calibration light source for the ground-based calibration of the TUS telescope. The angular distribution of the LED optical output power is illustrated in Fig.3 (left).
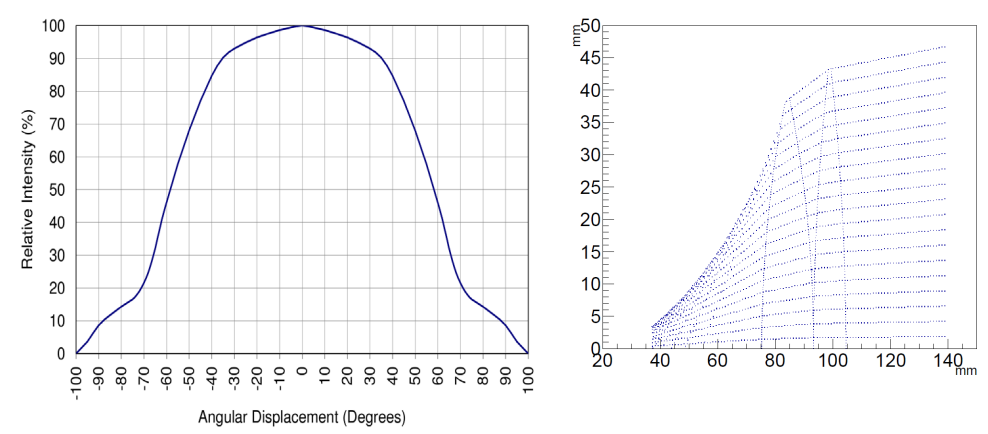

Figure 3: Typical radiation pattern of LedEngin-LZ4-00U600 LED(left). Schema of two lens collimation system(right).

As it is immediately obvious from this figure, despite the integrated lens the output the light flux is exceedingly wide and in this configuration, about $80 \%$ of the optical output power would be lost for the purpose of calibration. The solution to this problem, imposed 


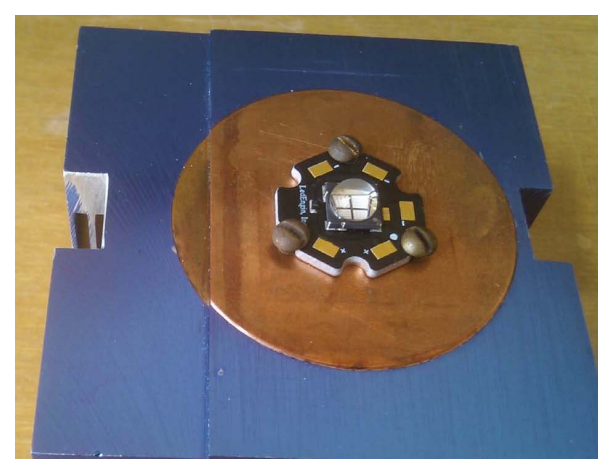

Figure 4: LedEngin-LZ4-00U600 LED together air cooler

by the cooling requirements for the LED (see Fig.4), is to use additional collimation lens (Fig.3, left) to reduce the angular width of the light flux to a value corresponding to $\pm 4.5^{\circ}$ FoV of the TUS telescope. Design and fabrication of such source prototype inluding the lens is under way.

\section{Conclusion}

The TUS mission aboard the dedicated Lomonosov satellite will be the first mission to use an orbital UV telescope for the study of EASs produced by UHECR. As such, it will provide important information and data for similar future missions such as MiniEUSO, JEM-EUSO and KLYPVE. During its $3 \div 5$ years operation, the exposure of the TUS telescope will be $12000 \div 20000 \mathrm{~km}^{2}$.sr, which is comparable with the exposure of the largest ground-based detectors. The ground-based calibration methods presented here are based on the use of lasers and high-power LEDs, and prototypes of the latter are currently under development.

Acknowledgement: The authors thank TUS collaborators G.Garipov. B.Khrenov, S. Sharakin and I.V. Yashin for supporting of the activity. This work was partially supported by RFBR research project No. 15-02-05498 A.

\section{References}

[1] B. A. Khrenov et al., Nucl. Phys. B (Proc. Suppl.) 112 (2002) 115.

[2] B. A. Khrenov et al., Phys. Atom. Nucl. 67(11) (2004) 2058.

[3] V. Abrashkin et al., Advances in Space Research 37 (2006) 1867.

[4] P. A. Klimov et al., 33d ICRC Proc. Rio De Janeiro (2013) ID 0406.

[5] L. Tkatchev et al., 30th ICRC Proc. Merida (2007). Vol.5, p.881, ID 0162.

[6] A. Tkachenko et al., 32d ICRC Proc. Beijing 11 (2011) ID 272.

[7] G. Garipov et al., Phys. Part. Nuclei Lett. 10(1) (2013) 49.

[8] A. Tkachenko et all., 33d ICRC Proc. Rio De Janeiro (2013) ID 0423.

[9] http://www.ledengin.com/files/products/LZ4/LZ4-00U600.pdf 